\title{
Mode de gouvernance des signes de qualité et comportements d'innovation
}

Une étude dans la région Languedoc-Roussillon

Governance patterns of quality signs and innovation behaviour in Languedoc-

Roussillon agro-food firms

\section{Fatiha Fort, Catherine Peyroux et Leila Temri}

\section{(2) OpenEdition}

\section{Journals}

Édition électronique

URL : http://journals.openedition.org/economierurale/1984

DOI : 10.4000/economierurale. 1984

ISSN : 2105-2581

Éditeur

Société Française d'Économie Rurale (SFER)

Édition imprimée

Date de publication : 30 décembre 2007

Pagination : 23-39

ISSN : 0013-0559

Référence électronique

Fatiha Fort, Catherine Peyroux et Leila Temri, « Mode de gouvernance des signes de qualité et comportements d'innovation », Économie rurale [En ligne], 302 | Novembre-décembre 2007, mis en ligne le 30 décembre 2009, consulté le 30 avril 2019. URL : http://journals.openedition.org/ economierurale/1984; DOI : 10.4000/economierurale.1984 


\title{
Mode de gouvernance des signes de qualité et comportements d'innovation Une étude dans la région Languedoc-Roussillon
}

\author{
Fatiha FORT • SupAgro, UMR Marchés, Organisations, Institutions, \\ Stratégies d'Acteurs (MOISA), Montpellier \\ Catherine PEYROUX • Équipe de recherche sur la firme et l'industrie (ERFI), \\ Université Paul Valéry Montpellier III \\ Leila TEMRI • Équipe de recherche sur la firme et l'industrie (ERFI), \\ Université Montpellier I, UFR Sciences économiques
}

\section{Introduction}

e secteur agroalimentaire est caractérisé - par deux tendances de fond. D'une part, les entreprises multiplient le recours aux signes de qualité, dont plusieurs font référence au territoire d'origine. D'autre part, le secteur n'échappe pas à l'innovation, considérée comme un moteur essentiel de la dynamique de croissance, à la fois du marché et des entreprises. Ces deux tendances sont le plus souvent considérées comme s'inscrivant dans une dynamique positive. Or, si l'on s'intéresse, dans le secteur agroalimentaire, à une certaine approche de la qualité, appréhendée à travers les modes de gouvernance des signes de qualité, et leur impact sur l'entreprise elle-même, cette vision nous paraît devoir être nuancée.

Nous supposons que les modes de gouvernance des signes de qualité, peuvent influencer plus ou moins favorablement les comportements d'innovation des entreprises engagées dans ces démarches de certification, en étant susceptibles d'engendrer des contraintes sur ces comportements. En nous inspirant de la définition des modes de gouvernance des entreprises de Perez (2003), par modes de gouvernance, nous entendons les dispositifs institutionnels et comportementaux régissant les relations entre les acteurs engagés dans une démarche de signalisation de la qualité. Il s'agit de contribuer à l'analyse de la dynamique qualité, innovation et territoire, initiée notamment par les travaux d'Allaire et Sylvander (1997), Sylvander et Marty (2000), ou encore de Touzard (2000). Mais ces auteurs se situent à l'échelle de systèmes de production, et sur une période de temps plus longue. Notre approche est centrée sur l'entreprise et son comportement d'innovation, et souligne l'incidence de l'engagement d'une entreprise dans une démarche de signalisation de la qualité sur son comportement d'innovation. Nous relierons le mode d'engagement de l'entreprise dans une démarche de signalisation à différentes formes de proximité, essentielles au processus d'innovation (Rallet et Torre, 2007), particulièrement dans le cas des petites entreprises (Freel, 2003). Notre hypothèse sera étudiée à partir des résultats de l'enquête INRA-PSDR ${ }^{1}$, réalisée en 2003 auprès d'un échantillon représentatif de 380 entreprises agroalimentaires de la région Languedoc-Roussillon.

Dans une première partie, nous développerons le cadre d'analyse, puis les résultats issus de l'enquête seront présentés et analysés.

\footnotetext{
1. Enquête sur les facteurs de développement des entreprises agroalimentaires du Languedoc-Roussillon, réalisée dans le cadre du programme de recherche « Pour et sur le développement rural » piloté par l'INRA.
} 


\section{Comportements d'innovation et signes de qualité}

La problématique examinée dans le cadre de cet article s'inscrit dans la question plus générale des relations entre innovation et qualité, qui sera ensuite déclinée en fonction de différents modes de gouvernance des signes de qualité, certains signes étant liés au territoire. Les modes de gouvernance des signes de qualité peuvent alors être analysés en termes de proximité.

\section{Innovation et qualité : quelle dynamique?}

Après avoir précisé l'approche de l'innovation retenue dans ce travail, nous présenterons les relations entre la qualité et l'innovation telles qu'elles apparaissent généralement dans la littérature.

Une approche de l'innovation large et relative Afin de rendre compte des innovations existantes dans le secteur agroalimentaire, il est nécessaire de se doter d'une définition qui permette de rendre compte de la diversité des situations innovantes rencontrées. C'est pourquoi nous avons privilégié une approche large et relative de l'innovation.

L'innovation peut de prime abord être définie comme l'introduction d'une nouveauté dans le champ économique. Les analyses récentes tendent à préciser cette définition en termes de processus d'apprentissage et de création de connaissances (Nonaka et Takeuchi, 1997 ; Divry et al, 1998), mettant en jeu des interactions entre différentes catégories d'acteurs (Akrich et al, 1988). Toutefois, la définition la

2. Citons pour mémoire: "Une innovation est la mise en ceuvre d'un produit (bien ou service) ou d'un procédé nouveau ou sensiblement amélioré, d'une nouvelle méthode de commercialisation ou d'une nouvelle méthode organisationnelle dans les pratiques de l'entreprise, l'organisation du lieu de travail ou des relations extérieures. » (OCDE, 2005, p. 56) plus opérationnelle, compte tenu de la méthodologie retenue, est celle de l'OCDE $(2005)^{2}$, dans sa version la plus récente. Elle permet tout d'abord de ne pas limiter l'innovation aux seuls domaines technologiques, mais d'inclure aussi celle dans les pratiques organisationnelles et commerciales. Elle comprend des innovations moins radicales, qui n'en constituent pas moins des sources de changement - changement identifié selon la perception des dirigeants le plus souvent - mais aussi d'amélioration des performances pour l'entreprise.

Par rapport à la typologie classique de l'OCDE, qui distingue innovation de produit, de procédé, commerciale et organisationnelle, nous avons préféré la notion d'innovation d'emballage à celle d'innovation marketing, car d'une part l'emballage est une source fondamentale d'innovations dans le secteur agroalimentaire, d'autre part, les modifications de l'emballage ou du design constituent la très grande majorité des innovations marketing, tandis que les autres types d'innovations marketing ou commerciales rencontrées peuvent être inclus dans l'innovation organisationnelle (Montaigne et Cadot, 2006 ; Ambiaud, 2007).

Le caractère innovant s'apprécie par rapport à un environnement qui va du niveau le plus local (celui de l'entreprise, de sa localisation dans une région) à un niveau plus global (son pays ou son marché mondial). Enfin, il est nécessaire d'inscrire dans le temps l'analyse de l'innovation. Le recours à la notion de processus le permet. Si, dans notre étude, du point de vue méthodologique, nous nous positionnons à un moment donné, plus précisément sur une courte période, pour observer la nature des innovations développées, nous nous inscrivons toutefois dans le cadre de processus d'innovation où il est parfois difficile de distinguer, par exemple, le lancement d'un nouveau produit d'une innovation commerciale ou organisationnelle qui en est la conséquence (Belotti et Temri, 2004). 
La qualité et ses relations à l'innovation La qualité a fait l'objet d'une abondante littérature, et de nombreuses définitions qui nous semblent inutiles de reprendre intégralement. Valceschini et Nicolas (1995) relèvent que dans l'agroalimentaire, elle peut désigner soit un niveau de performance supérieur, soit une propriété permettant de distinguer, d'identifier le produit, soit, dans l'acception de $\mathrm{l}^{\prime} \mathrm{AFNOR}{ }^{3}$, son aptitude à satisfaire les besoins des utilisateurs. Les modalités de gestion de la qualité se sont largement focalisées sur l'analyse et la mise au point d'outils et de démarches destinés à l'entreprise. Des démarches collectives ont été plus particulièrement développées et analysées dans l'agriculture et l'agroalimentaire. Le sens de la relation entre qualité et innovation est controversé. Prajogo et Sohal (2001) ont réalisé une synthèse des arguments, dans le cadre de procédures de TQM (Total Quality Management). Les principes du TQM seraient, pour certains auteurs, congruents avec ceux de l'innovation, donc favorables à l'innovation, quelle que soit sa nature. Des études empiriques ont confirmé ces réflexions, et certains, tels Mc Adam et al. (1998) suggèrent que l'introduction de pratiques de management de la qualité stimule à terme l'innovation. Roux (1999) a relevé que dans l'agroalimentaire, le management de la qualité se traduit par des changements organisationnels. D'autres travaux suggèrent que l'entrée de l'entreprise dans une démarche d'amélioration de la qualité peut entraîner une innovation organisationnelle, car elle nécessite souvent une remise à plat des processus de production et une réorganisation des relations amont/aval (Fort et Fort, 2006). À une autre échelle d'analyse, une confusion des termes est parfois opérée. Ainsi Touzard (op. cit.) appréhende la transformation de la viticulture languedocienne d'une production de masse vers des vins de qualité, comme un processus d'innovation réalisé en interaction avec

3. Association française de normalisation. les territoires. Allaire et Sylvander (op. cit.) assimilent qualité et innovation, en considérant l'amélioration de la qualité comme l'une des principales directions prises par l'évolution des systèmes d'innovation agroalimentaires. Ainsi, de nombreux exemples attestent de l'existence d'une relation positive, un « cercle vertueux », entre innovation et démarche de qualité.

Mais d'autres travaux récusent cette relation positive. Selon Prajogo et Sohal (op. cit.), certains auteurs avancent que le TQM favorise l'innovation incrémentale au détriment de la recherche d'innovations radicales. De plus, ils observent que les démarches qualité peuvent conduire à terme à une standardisation des processus et procédures, à l'instauration de routines néfastes à l'innovation, car pour l'entreprise, la démarche qualité s'accompagne également de la nécessité de respecter un certain nombre de règles, et de standardiser certains éléments de son processus ou de ses produits. Ces contraintes ne risquent-elles pas de limiter l'innovation?

Les signes de qualité :

un frein à l'innovation?

Dans l'agroalimentaire la maîtrise de la qualité et sa valorisation nécessitent des coordinations qui dépassent parfois l'unité de production pour englober les filières, à travers différentes procédures (Valceschini et Nicolas, op. cit.). Un dispositif de signalisation de la qualité et de l'origine a été mis en place progressivement en France, autour de signes officiels. Ce dispositif a ensuite été relayé au niveau de l'Europe, qui s'est inspirée de la réglementation française, et a connu des évolutions récentes en France. Un dispositif récent distingue les signes officiels de qualité et d'origine, les mentions valorisantes, et les démarches de certification des produits. Dans la première catégorie, sont distingués les signes par lesquels la qualité est liée à l'origine et à la tradition, ceux attestant d'une qualité supérieure, et enfin ceux traduisant une qualité 
environnementale. L'attribution de ces signes, est fortement réglementée, et la rédaction des cahiers des charges, pour chaque demande de reconnaissance, encadre les comportements des entreprises. Chaque cahier des charges est élaboré selon un processus qui dépend des modes de gouvernance de chaque type de signe de qualité, et peut se traduire, au final, par des contraintes plus ou moins fortes pour les entreprises engagées ${ }^{4}$. Les modes de gouvernance des signes de qualité peuvent être analysés en termes de proximité, comme nous le détaillerons dans la section suivante. Les contraintes engendrées ne risquent-elles pas de bloquer l'innovation? Il peut être ainsi difficile de faire évoluer le produit ou le processus de production sans sortir d'un cahier des charges inspiré des pratiques ancrées dans l'histoire, la tradition et les conditions naturelles d'un territoire.

Ainsi, Roux (op. cit.), dans une étude sur les entreprises agroalimentaires, note que les entreprises dites en « gestion traditionnelle », c'est-à-dire celles peu engagées dans des mutations organisationnelles ou managériales, et dans la mise en place de réseaux informatiques, sont souvent des entreprises déjà engagées dans les démarches "d'appellation d'origine » ou « de terroir ». Les entreprises liées au terroir seraient ainsi moins innovantes en organisation. Inversement, d'autres signes de qualité intègrent les possibilités d'innovation dans les critères mêmes de labellisation, notamment à travers des notices techniques nationales.

Ce constat nous amène à préciser davantage la relation entre signe de qualité et comportement d'innovation, en fonction des modes de gouvernance des signes de qualité.

\section{Modes de gouvernance et signes de qualité}

Le territoire suscite des modes de gouvernance particuliers des signes de qualité ;

4. Par exemple, l'interdiction d'utiliser du lait caillé dans l'AOC Pélardon. mais d'autres logiques, plus sectorielles, peuvent apparaître. L'intégration du degré de contrainte dans l'analyse nous conduira à proposer une typologie des modes de gouvernance des signes de qualité, qui sera reliée à différentes formes de proximité. Depuis les années 1980-1990, la proximité fait en effet l'objet de nombreuses analyses dans le cadre des processus d'innovation (Rallet et Torre, op. cit.). Dans notre cas, l'analyse des liens entre qualité et innovation nous renvoie à une analyse en termes de proximité par l'intermédiaire des modes de gouvernance des signes de qualité.

\section{Modes de gouvernance des signes de qualité}

De nombreux signes de qualité existent dans le dispositif national et européen. Chacun d'eux est doté d'un mode de gouvernance particulier qui régit son fonctionnement, les relations entre les acteurs qui établissent un cahier des charges puis le mettent en œuvre, ainsi que les relations de ces derniers avec les organismes chargés du contrôle. Allaire et Sylvander, puis Sylvander et Marty distinguent deux types de gouvernance des signes de qualité : une gouvernance territoriale et une gouvernance sectorielle.

Le territoire contribue au développement de ressources spécifiques, en favorisant la coordination des acteurs et des coopérations plus horizontales que verticales. Cette forme de gouvernance est particulièrement liée aux institutions régionales ou nationales. Elle s'appuie sur des démarches collectives, fondées sur une logique de coopération entre acteurs institutionnels, représentants professionnels et entreprises. Delfosse et Letablier (1995) ont ainsi mis en évidence des processus de construction de systèmes de qualité spécifiques associés à des territoires, dans le cadre d'organisations territorialisées. Le lien au territoire atteste d'une proximité spatiale entre les acteurs, et celle-ci est doublée d'une proximité organisationnelle mais aussi institutionnelle : les acteurs engagés collective- 
ment dans un signe de qualité à gouvernance territoriale se coordonnent à travers des représentations et des règles de fonctionnement communes, fondées d'ailleurs sur les spécificités du territoire, de l'espace géographique et social. Ils sont en interaction autour d'un projet commun, et définissent leurs stratégies et leurs comportements dans le cadre de ces règles communes (Pecqueur et Zimmermann, 2004). Dans le cas de la région Languedoc-Roussillon, une gouvernance territoriale de ce type existe. Elle est souvent associée à la filière vitivinicole (Touzard, op . cit.). Dans cette filière, l'existence de réseaux de producteurs déjà constitués a favorisé l'émergence d'une gouvernance territoriale, très largement coordonnée par les institutions régionales ${ }^{5}$, qui a contribué à la dynamique d'innovation de la viticulture du Languedoc-Roussillon vers les productions de qualité.

À cette dimension territoriale, il est possible de combiner une dimension sectorielle, issue d'une coopération fondée sur le domaine d'activité lui-même, avec ses normes et ses règles de concurrence et de coordination propres (Sylvander et Marty, op. cit.). Les normes sectorielles sont élaborées institutionnellement, sans lien particulier avec le territoire. La viticulture ellemême semble combiner les deux logiques. Dans ce cas, la proximité géographique n'est pas fondamentale, tandis que les proximités institutionnelle et organisationnelle marquent les modes de coordination entre les individus.

Ces modes de gouvernance à la fois territoriale et sectorielle permettent donc d'exploiter des proximités géographiques, institutionnelles et organisationnelles qui semblent jouer un rôle moteur dans les innovations et les performances économiques.

5. Activité importante de la société Prodexport pour le compte de la Région, de la Direction régionale du commerce extérieur et de la COFACE pour le compte de l’État.

\section{Signes de qualité et lien au territoire}

De nombreux travaux ont traité du rôle du local dans la définition et la gestion de la qualité des produits alimentaires, en particulier dans les cas de l'AOC/AOP et l'IGP (Casabianca et Valceschini, 1996). L'analyse de la structure de l'offre de ces produits de qualité territoriale (Mollard, 2001) fait apparaître différentes formes d'action collective et de coordination des acteurs dans la gestion du bien commun que représente le signe de qualité (Tirole, 1996).

Pour le consommateur, la référence à l'origine est souvent interprétée comme un gage de qualité. Les produits d'origine sont perçus comme traditionnels, authentiques, résultats de savoir-faire locaux. Ils sont effectivement construits localement par interaction entre production et consommation et destinés à un marché au départ local, puis progressivement national et même international.

Les producteurs, identifiant une opportunité commerciale, vont rechercher l'obtention de cette labellisation territoriale, ce qui relève d'une démarche souvent collective dans laquelle les institutions locales et professionnelles sont activement impliquées. Cette référence au territoire est aussi un moyen de différencier les produits et de les protéger.

La mise en place d'une réglementation européenne depuis 1992, concernant la protection de l'origine géographique des produits, a encouragé et accompagné de telles initiatives collectives ou individuelles. L'arsenal législatif européen repose sur trois signes de qualité :

- l'AOP (Appellation d'origine protégée), dont la philosophie est de protéger un produit unique, non reproductible dans un autre terroir. La totalité du processus de la production (origine de la matière première locale et fabrication sur place) doit s'y dérouler ;

- l'IGP (Indication géographique protégée) basée principalement sur la réputation du produit, sur son histoire, qui lui confère des 
qualités particulières (la matière première n'est pas obligatoirement originaire de la région) ;

- la STG (Spécialité traditionnelle garantie), ou Attestation de spécificité, elle, protège une tradition. Le produit doit présenter une certaine spécificité qui le distingue d'autres produits ou denrées similaires. Aucune obligation d'origine géographique n'est mentionnée.

En France, cette réglementation, nouvelle pour plusieurs pays, est venue renforcer un arsenal juridique déjà bien étoffé en signes de qualité. À côté de l'AOC (qui a inspiré l'AOP européenne), on trouve le Label rouge, attestant d'une qualité supérieure du produit, et le Certificat de conformité produit (CCP) de création plus récente (1990). Aucun lien entre le produit et un terroir n'est obligatoire dans ces deux signes de qualité ; cependant dans leurs cahiers des charges respectifs, il est possible de mettre en avant un lieu de production et de prétendre ainsi à une IGP.

Les signes de qualité que nous venons de présenter sont liés au produit, et éventuellement au processus de production. D'autres signes de qualité ne concernent que les processus de production comme l'Agriculture biologique $(\mathrm{AB})$. Ces signes de qualité n'apparaissent pas liés au territoire.

On constate une grande diversité dans les formes de coordination locales des signes de qualité qui ne relèvent pas tous d'une gouvernance territoriale. Des exigences sectorielles peuvent aussi conduire à l'adoption de signes de qualité. Ont-ils tous la même influence sur les pratiques innovantes ?

\section{Typologie des modes de gouvernance}

Les différents signes de qualité se basent tous sur des cahiers des charges plus ou moins contraignants. Nous avons cherché à évaluer le degré de contraintes vis-à-vis de l'innovation, lié au mode de gouvernance de ces signes de qualité.

Afin de classer les différents signes de qualité en fonction de leurs modes de gou- vernance, nous avons retenu deux critères.

Le premier, dans la logique des travaux d'Allaire et Sylvander (op. cit.) correspond au caractère territorialisé ou non des modes de gouvernance. La non-territorialisation renvoie en fait à la prédominance d'un mode de gouvernance sectorielle, marquée par les proximités institutionnelle et organisationnelle. Selon ces auteurs, le Label rouge apparaît fortement lié à une gouvernance sectorielle, tandis que l'AOC et l'IGP sont le plus souvent très dominés par une forme de gouvernance territoriale, où la proximité spatiale est une condition indispensable.

Le deuxième critère retenu est celui du caractère collectif ou individuel de ce mode de gouvernance. Car si certains signes de qualité ne peuvent être demandés que dans le cadre d'une démarche collective (AOC ou Label rouge par exemple), d'autres correspondent à des démarches volontaires individuelles (CCP, AB). Dans le premier cas, nous retrouvons les proximités organisationnelle et institutionnelle, éventuellement géographique, tandis que dans le second cas, si les entreprises peuvent développer leurs propres réseaux, la proximité n'est pas formalisée obligatoirement dans des règles de fonctionnement liant indissociablement les acteurs engagés. Les modes de coordination sont plus souples, et les liens sont plus facilement réversibles parce qu'il n'existe pas d'organisation «proche », au sens relationnel, qui les institutionnalise. Ce deuxième critère, croisé avec le précédent, permet d'obtenir le tableau 1 .

Les axes de la matrice ainsi obtenue peuvent faire l'objet d'une interprétation en termes de contrainte par rapport aux comportements d'innovation. Ainsi les modes de gouvernance territoriaux tout comme les modes collectifs peuvent se révéler fort contraignants.

Le lien au territoire différencie et protège le produit, mais peut générer des contraintes, non seulement en termes de localisation de la production mais également sur le produit, le processus de pro- 


\begin{tabular}{|c|c|c|c|}
\hline \multicolumn{3}{|c|}{ contraintes } & \multirow{3}{*}{$\uparrow+$} \\
\hline+ & & & \\
\hline & Collectifs & Individuels & \\
\hline Territorialisés & $\begin{array}{c}\text { AOC/AOP, IGP , STG } \\
\text { marques collectives } \\
+++\end{array}$ & $\begin{array}{c}\text { Marques propres } \\
\text { comportant un } \\
\text { nom de lieu } \\
+ \\
\end{array}$ & contraintes \\
\hline Non Territorialisés & $\begin{array}{c}\text { Label rouge, } \\
++\end{array}$ & $\begin{array}{c}C C P, A B \\
+ \\
\end{array}$ & - \\
\hline
\end{tabular}

duction, ou les approvisionnements, qui doivent être explicités précisément, en fonction des particularités physiques et sociales du territoire, et peuvent ainsi être figés sans possibilité d'évolution.

Le caractère plus ou moins collectif des démarches de qualification peut lui aussi s'interpréter comme un indicateur du degré de contrainte. Si les démarches collectives de qualification présentent un grand nombre de bénéfices et conduisent à une amélioration de la qualité à l'échelle de la collectivité, elles résultent de négociations longues et difficiles entre les acteurs et peuvent déboucher sur des contraintes collectives fortes. Elles rendent possibles les actions collectives, mais en même temps, elles orientent les comportements des acteurs, et circonscrivent ainsi "leur liberté et leur capacité d'action »(Crozier et Friedberg, 1977) ${ }^{9}$ Elles peuvent amener à une standardisation de la production, qui signifie uniformisation et réduction de la diversité, s'opposant ainsi à l'innovation (Foray, 1996). Dans le cas de signes individuels, chaque entreprise est seule responsable de son propre cahier des

6. La marque propre n'est pas un signe officiel de qualité. Nous la mentionnons cependant dans la mesure où elle représente un signe distinctif susceptible d'être territorialisé (exemple la grisette de Montpellier ou les vignerons catalans).

7. Par exemple, produits du Pays Cathare dans l'Aude.

8. Dans cet article, les sources des tableaux et histogrammes sont issues des calculs des auteurs.

9. $C f$. page 16 . charges, et de sa modification éventuelle.

Les signes individuels de qualité sont ici considérés comme moins contraignants que les signes collectifs, tout comme les signes de qualité non territorialisés. Cela signifierait que les signes individuels et non territorialisés sont les moins contraignants pour le producteur, suivis par les signes individuels et non territorialisés, ainsi que les signes collectifs et non territorialisés, tandis que les signes collectifs et territorialisés sont ceux qui laissent le moins de marge de manœuvre.

Ainsi, si qualité et innovation sont le plus souvent considérées comme associées positivement, certaines observations tendent à établir une liaison négative, notamment dans le cas de signes de qualité liés au territoire. Pour approfondir l'étude de cette hypothèse, nous avons ajouté à la dimension territorialisée ou non des signes de qualité un second axe, le caractère collectif ou non de la démarche de qualification. L'appréciation des modes de gouvernance en fonction du degré de contraintes nous permet ainsi de préciser notre hypothèse initiale.

Ces propositions sont-elles validées par les résultats des enquêtes réalisées dans le cadre du programme PSDR en LanguedocRoussillon?

\section{Résultats de l'étude empirique}

Les propositions ont été confrontées aux résultats de l'enquête INRA-PSDR concernant les comportements d'innovation et les signes de 
qualité. Après avoir présenté la méthodologie de l'enquête réalisée et les principales caractéristiques des variables étudiées, nous procèderons à l'analyse des relations entre différents signes de qualité et le comportement en matière d'innovation des entreprises.

\section{Comportements des entreprises agroalimentaires en matière $d^{\prime}$ 'innovation et de signes de qualité}

Préalablement à la présentation des principaux résultats concernant chacune des deux variables étudiées, quelques indications méthodologiques sont fournies.

\section{Précisions méthodologiques}

Les résultats présentés dans ce paragraphe sont issus de l'enquête INRA-PSDR, réalisée en 2003 auprès des entreprises agroalimentaires de la région Languedoc Roussillon. La base de données comporte 339 entreprises représentatives de 1073 entreprises agroalimentaires de la région). Le traitement des données a été réalisé par le logiciel SPSS et se base sur les données extrapolées en fonction du plan de sondage (Agreste, 2004).

L'agroalimentaire régional est dominé par la filière vin, qui représente $43 \%$ des établissements, et totalise $46 \%$ du chiffre d'affaires, suivi par les dérivés des céréales (24\% du nombre d'établissements et $13 \%$ du chiffre d'affaires), les fruits et légumes (10\% du nombre d'établissements et $14 \%$ du chiffre d'affaires) et enfin les produits animaux (14\% du nombre d'établissements et $14 \%$ du chiffre d'affaires).

Nous nous sommes intéressées ici à l'étude de deux variables, le comportement d'innovation des entreprises d'une part, et les différents signes de qualité utilisés par l'entreprise pour son produit principal, d'autre part.

La variable comportement d'innovation a été appréhendée à travers quatre types d'innovations (l'innovation de produit, l'innovation de procédé, l'innovation d'emballage et/ou conditionnement et l'innovation organisationnelle) $)^{10}$, que l'entreprise a réalisés au cours des trois dernières années. Les réponses obtenues sont fondées sur l'appréciation personnelle du chef d'entreprise.

Pour la variable signes de qualité utilisés par l'entreprise, le chef d'entreprise a mentionné ceux qui étaient effectivement associés à son produit principal.

\section{Analyse descriptive des variables}

L'analyse des résultats nous permet de caractériser le comportement des chefs d'entreprises interrogés en matière d'innovation, et les signes de qualité qu'ils ont adoptés.

\section{- Le comportement d'innovation}

Dans l'échantillon, tous les types d'innovation ont été mis en œuvre. Au total, $54,3 \%$ des entreprises ont innové sur au moins une des composantes. Ce chiffre est à rapprocher de celui de la dernière enquête nationale, qui fait état de près de $60 \%$ d'entreprises agroalimentaires de plus de 10 salariés innovantes sous au moins une forme entre 2002 et 2004 (Ambiaud, op. cit.).

On constate par ailleurs un pourcentage d'entreprises innovantes différent selon le type d'innovation (histogramme 1) : près de $40 \%$ des entreprises interrogées ont déclaré avoir innové au moins une fois dans le produit au cours des trois dernières années (contre $27 \%$ dans l'emballage et le conditionnement, $20 \%$ dans les procédés et $23 \%$ dans l'organisation de l'entreprise).

L'histogramme montre que l'innovation de produit est la plus fréquente. Un examen plus approfondi des résultats indique qu'il existe une forte corrélation entre les types d'innovation: les entreprises innovent souvent selon plusieurs modalités (tableau 2).

10. La question était formulée ainsi : «Au cours des 3 dernières années, avez-vous réalisé : au moins une innovation de produit/au moins une innovation de procédélau moins une innovation emballage et/ou conditionnement/au moins une innovation organisationnelle ?»... 


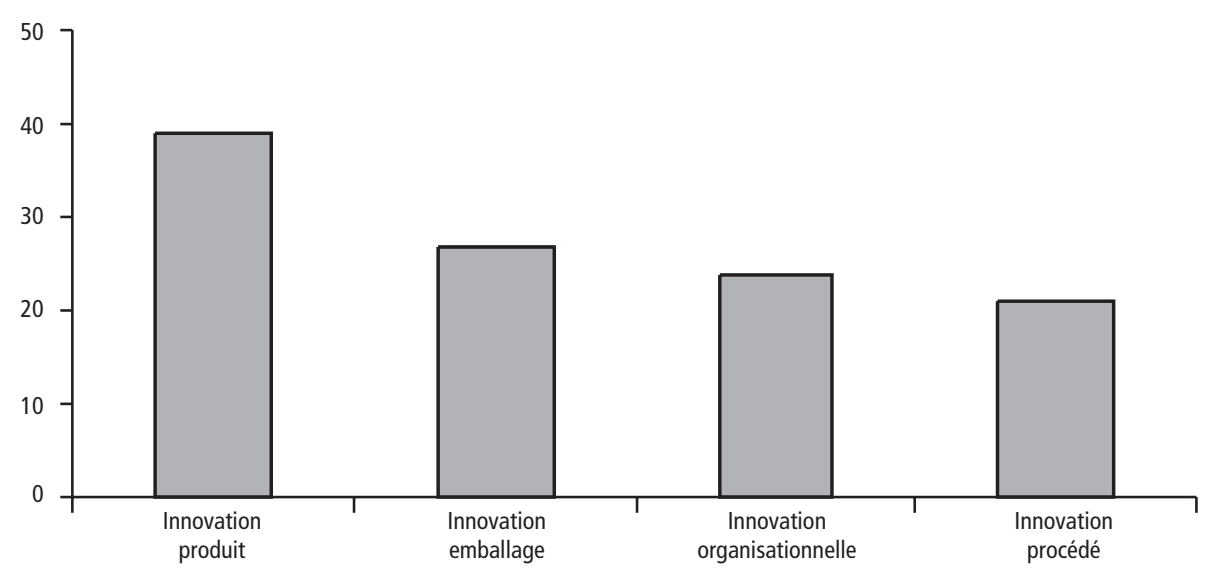

Tableau 2. Corrélation de Pearson entre les différents domaines d'innovation

\begin{tabular}{l|c|c|c|c}
\hline & $\begin{array}{c}\text { Innovation } \\
\text { produit }\end{array}$ & $\begin{array}{c}\text { Innovation } \\
\text { procédé }\end{array}$ & $\begin{array}{c}\text { Innovation } \\
\text { emballage }\end{array}$ & $\begin{array}{c}\text { Innovation } \\
\text { organisationnelle }\end{array}$ \\
\hline Innovation produit & 1 & & & \\
\hline Innovation procédé & $0,37^{* *}$ & 1 & & \\
\hline Innovation emballage & $0,387^{* *}$ & $0,187^{* *}$ & 1 & 1 \\
\hline Innovation organisationnelle & $0,345^{* *}$ & $0,464^{* *}$ & $0,29^{* *}$ & 1 \\
\hline
\end{tabular}

** La corrélation est significative au niveau 0,01 (bilatéral)

Si les quatre domaines d'innovation apparaissent comme fortement corrélés, la corrélation entre l'innovation procédé et l'innovation organisationnelle est plus conséquente que celle existant entre l'innovation procédé et l'innovation produit. Ce résultat rejoint ceux que nous avons constatés dans d'autres travaux (Fort et al, 2005 ; Ayerbe, 2006).

Remarquons cependant que cette dynamique de l'innovation (stimulation intertypes d'innovation) ne concerne qu'une entreprise sur deux. En effet, près de $46 \%$ des entreprises n'ont pas innové au cours des trois dernières années alors que $7 \%$ des entreprises ont innové selon les quatre modalités retenues. Ces chiffres peuvent un peu surestimer le recours à l'innovation dans la mesure où celle-ci est appréciée à partir des déclarations des dirigeants et donc de leur représentation de l'innovation.

Nous avons réalisé une analyse du comportement innovateur des entreprises en fonction de leur taille (histogramme 2). De nombreux travaux ont en effet montré l'impact de cette variable sur les comportements d'innovation (Pamukçu et Cincera, 2001 ; Bhattacharya et Bloch, 2004). Nous observons ici un fort effet de cette variable : $58 \%$ des entreprises de plus de 20 salariés ont innové contre $31 \%$ pour celles de 3 à 5 salariés. Les enquêtes nationales aboutissent au même constat (Ambiaud, op. cit.), avec notamment un taux d'innovation de $100 \%$ parmi les entreprises de plus de 1000 salariés (Vincenau, 2003).

Cet effet de la taille se retrouve dans toutes les composantes de l'innovation. Cependant on peut remarquer que chez les plus petites entreprises de l'échantillon (de 3 à 5 salariés), l'innovation organisationnelle est relativement plus importante que dans les entreprises de plus grande taille (de 6 à 9 salariés). Cette particularité peut s'expliquer par l'engagement de ces entreprises, notamment les caves particulières qui repré- 
Histogramme 2. Type d'innovation et taille d'entreprise (en \% de la population totale)

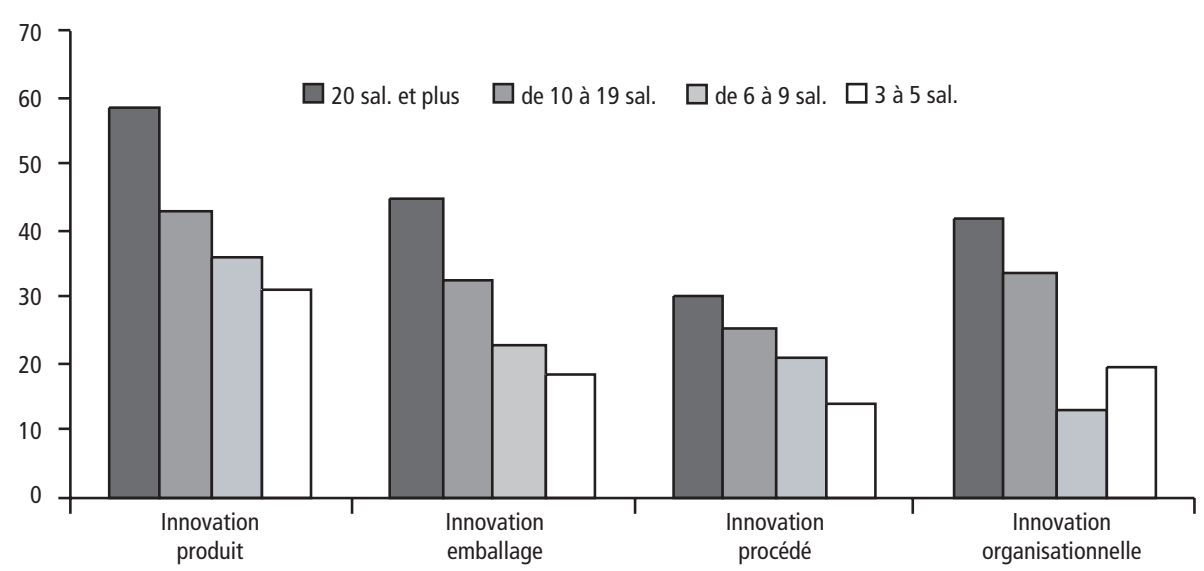

sentent plus de $60 \%$ de ces petites entreprises, dans des procédures de certification de la qualité et de réorganisation commerciale.

De la même façon, les domaines d'innovation semblent différents selon le secteur d'activité (histogramme 3). Le choix de cette variable sectorielle s'inscrit dans le prolongement des travaux pionniers de Pavitt (1984), et s'inspire, dans le domaine agroalimentaire, de nombreuses observations empiriques.

Si globalement l'innovation sur le produit apparaît comme la plus importante dans les différentes filières, on peut noter la forte implication des entreprises de la filière fruits et légumes dans l'innovation d'emballage du produit compte tenu de la nature du produit (souvent des produits frais).

- Le recours aux signes de qualité

En matière d'adoption de signes de qualité, nous avons étudié les signes de qualité du principal produit des entreprises, produit qui représente en moyenne $83 \%$ du chiffre d'affaires (histogramme 4).

Si $40 \%$ des entreprises n'ont aucun signe de qualité, l'AOC et les autres dénominations d'origine concernent plus de la moitié des entreprises. L'AOC domine, suivie des autres dénominations géographiques (généralement les Vins de Pays, les labels régionaux...).

Histogramme 3. Les différents types d'innovation par secteur (en \% de la population totale)

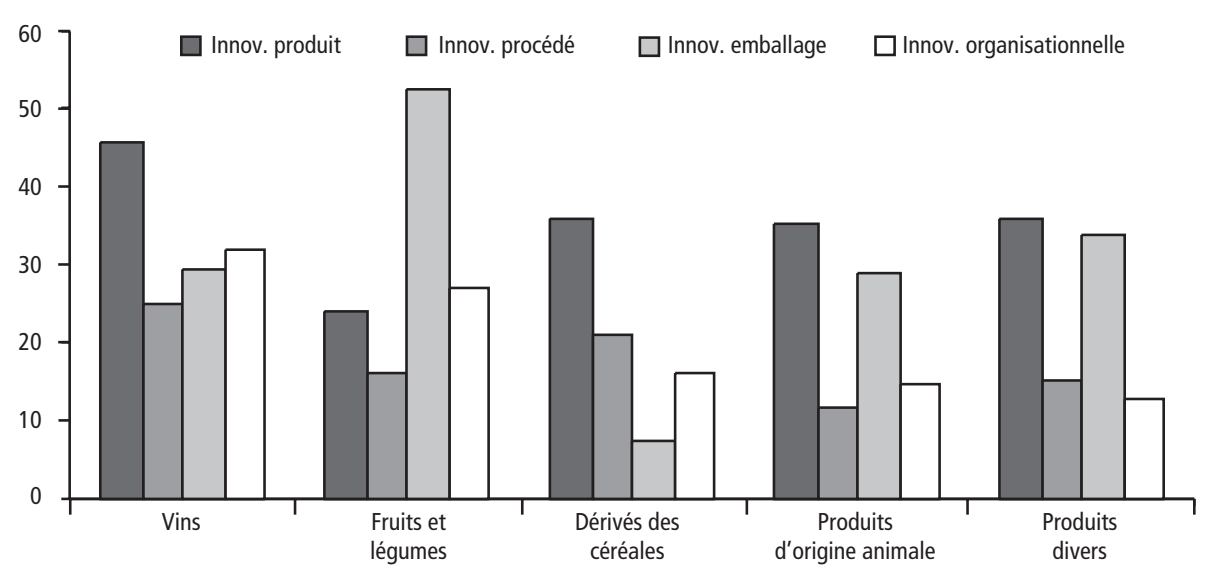




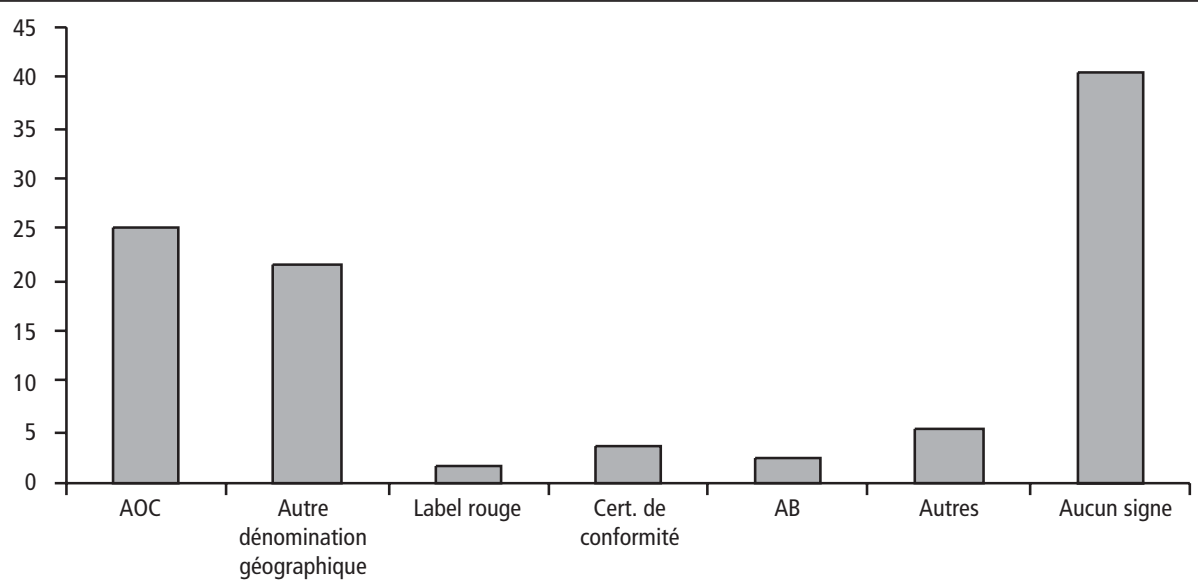

L'analyse de l'importance des signes de qualité par secteur (tableau 3) montre que la filière vin est dominée par les signes de qualité liés au terroir (AOC et autres dénominations géographiques) alors que dans les autres filières, on constate soit, le plus fréquemment, l'absence de signes de qualité, soit la présence dominante de signes non liés au territoire (Label rouge, Certificat de conformité produit, - CCP - ou Agriculture biologique, $-\mathrm{AB}-$ ). De même, les signes de qualité territoriaux, AOC et autres dénominations géographiques, sont constitués à plus de $90 \%$ d'entreprises appartenant à la filière vin, tandis que les signes non territoriaux, Label rouge, $\mathrm{AB}$ et $\mathrm{CCP}$ sont représentés par les dérivés de céréales, les produits d'origine animale et, dans une moindre mesure, par les fruits et légumes.

Cet effet sectoriel dans le choix des signes de qualité est important, comme on peut le remarquer dans le tableau 3. Il est particulièrement marqué par l'association AOC et autres dénominations géographiques avec les entreprises du secteur viticole. $44,4 \%$ des entreprises ayant adopté un signe de qualité appartiennent au secteur vin.

\section{Signes de qualité adoptés et comportements d'innovation}

Après avoir caractérisé le comportement innovateur des entreprises et les principaux signes de qualité présents dans les produits des entreprises, nous avons cherché à

Tableau 3. Signes de qualité et filières

\begin{tabular}{|c|c|c|c|c|c|c|c|c|c|}
\hline Filière & SQ & $\mathrm{AOC}$ & $\begin{array}{c}\text { Autre } \\
\text { dén. géo }\end{array}$ & $\begin{array}{l}\text { Label } \\
\text { rouge }\end{array}$ & $\begin{array}{l}\text { Certif. } \\
\text { conf. }\end{array}$ & $A B$ & Autres & Aucun & Tota \\
\hline \multirow[t]{2}{*}{ Vin } & $\%$ ds filière & 51,8 & 43,6 & 0 & 0 & 0,6 & 0,2 & 3,8 & 100 \\
\hline & $\%$ ds SQ & 91,4 & 90,8 & 0 & 0 & 11,5 & 1,8 & 4,1 & 44,4 \\
\hline \multirow[t]{2}{*}{ F\&L } & $\%$ ds filière & 4,5 & 1,8 & 0 & 0,9 & 9 & 20,7 & 63,1 & 100 \\
\hline & $\%$ ds SQ & 1,9 & 0,9 & 0 & 2,7 & 38,5 & 41,8 & 16 & 10,4 \\
\hline \multirow{2}{*}{$\begin{array}{l}\text { Dérivés } \\
\text { Céréales }\end{array}$} & $\%$ ds filière & 0 & 2,3 & 4,6 & 4,2 & 1,2 & 9,6 & 78,1 & 100 \\
\hline & $\%$ ds SQ & 0 & 2,6 & 66,7 & 29,7 & 11,5 & 45,5 & 46,5 & 24,3 \\
\hline \multirow{2}{*}{$\begin{array}{l}\text { Produits } \\
\text { Orig. Anim. }\end{array}$} & $\%$ ds filière & 4,3 & 9,3 & 4,3 & 17,1 & 3,6 & 3,6 & 57,9 & 100 \\
\hline & $\%$ ds SQ & 2,2 & 5,7 & 33,3 & 64,9 & 19,2 & 9,1 & 18,5 & 13,1 \\
\hline \multirow{2}{*}{$\begin{array}{l}\text { Produits } \\
\text { Divers }\end{array}$} & $\%$ ds SQ & 14,3 & 0 & 0 & 1,2 & 6 & 1,2 & 77,4 & 100 \\
\hline & $\%$ ds filière & 4,5 & 0 & 0 & 2,7 & 19,2 & 1,8 & 14,9 & 7,9 \\
\hline \multirow[t]{2}{*}{ Total } & & 25,1 & 21,3 & 1,7 & 3,5 & 2,4 & 5,1 & 40,8 & 100 \\
\hline & & 100 & 100 & 100 & 100 & 100 & 100 & 100 & 100 \\
\hline
\end{tabular}


Tableau 4. Signes de qualité et types d'innovation (en \% de la population totale)

\begin{tabular}{l|c|c|c|c|c|c|c|c}
\hline & AOC & $\begin{array}{c}\text { Autres dénom. } \\
\text { géog. }\end{array}$ & $\begin{array}{c}\text { Label } \\
\text { rouge }\end{array}$ & CCP & AB & Autres & Aucun & Total \\
\hline Innovation produit & 47,4 & 46,5 & 70,6 & 42,1 & 44 & 25 & 30 & 39,1 \\
\hline Innovation procédé & 24,8 & 21,7 & 55,6 & 13,5 & 36 & 16,1 & 17,1 & 20,9 \\
\hline Innovation emballage & 38,7 & 27,8 & 23,5 & 21,6 & 56 & 26,8 & 17,9 & 26,9 \\
\hline Innovation organisation & 34,8 & 27 & $\mathbf{5 5 , 6}$ & 15,8 & 15,4 & 28,6 & 15 & 24 \\
\hline
\end{tabular}

croiser ces deux variables afin de vérifier notre hypothèse concernant le lien négatif entre innovation et certains signes de qualité. Nous présentons tout d'abord les principaux résultats de ce traitement, puis les discutons.

\section{Les liens entre signes de qualité et comportement d'innovation}

Les résultats du croisement des variables « comportement d'innovation » et « signes de qualité » sont présentés dans le tableau 4. À titre d'illustration, 70,6\% des entreprises en Label rouge ont innové dans le produit, $55,6 \%$ dans l'organisationnel, alors que seulement $30 \%$ des entreprises sans aucun signe de qualité ont innové dans le produit.

Ce tableau suscite plusieurs commentaires :

1. Les entreprises ayant adopté un signe de qualité, quel qu'il soit, apparaissent incontestablement plus dynamiques, du point de vue de l'innovation, que celles sans signe de qualité. Ainsi, le pourcentage d'entreprises innovantes parmi celles sans signe de qualité est systématiquement en dessous de celles ayant un signe de qualité, sauf pour l'innovation procédé et, de manière plus marginale, produit (le pourcentage d'entreprises ayant un « autre » signe de qualité est plus faible que pour « aucun » signe de qualité dans l'innovation produit). Par ailleurs, les meilleurs scores d'innovation produit, procédé et organisation sont associés au Label rouge, et l'innovation emballage à l'Agriculture biologique. Ces résultats tendent à confirmer l'idée généralement admise qu'adoption de signe de qualité et innovation vont de pair.

2. Sur la dimension signe territorialisé/non territorialisé, deux signes non territorialisés, le Label rouge et l'AB, apparaissent comme plus innovants sur toutes les composantes de l'innovation. Cependant, la Certification de conformité, CCP, apparait globalement comme moins innovante. Si l'on agrège séparément signes territorialisés d'une part - AOC et « autres indications géographiques -, et non territorialisés d'autre part - Label rouge, $\mathrm{CCP}$, et $\mathrm{AB}$ - (tableau 5), on constate que les signes non territorialisés sont plus fréquemment innovants pour l'innovation produit et procédé, mais moins sur les dimensions emballage et surtout organisationnelle. Ainsi, par exemple, 49,4\% des entreprises sous signe non territorialisé ont innové en produit, contre $47 \%$ pour les signes territorialisés, et $30 \%$ des entreprises sans signe de qualité.

Tableau 5. Innovation et dimension territorialisée/non territorialisée des signes de qualité

\begin{tabular}{l|c|c|c|c|c}
\hline & SQ territ. & SQ non territ. & Autres & Aucun & Total* \\
\hline Innovation produit & $47 \%$ & $49,4 \%$ & $25 \%$ & $30 \%$ & $39,1 \%$ \\
\hline Innovation procédé & $23,2 \%$ & $30 \%$ & $16,1 \%$ & $17,1 \%$ & $20,8 \%$ \\
\hline Innovation emballage & $33,7 \%$ & $33,3 \%$ & $26,8 \%$ & $17,9 \%$ & $27 \%$ \\
\hline Innovation organisationnelle & $31,3 \%$ & $23,5 \%$ & $28,6 \%$ & $15 \%$ & $23,9 \%$ \\
\hline
\end{tabular}

*Plusieurs réponses possibles 
Tableau 6. Innovation et dimension collective/individuelle des signes de qualité

\begin{tabular}{l|c|c|c|c|c}
\hline & SQ coll. & $\begin{array}{c}\text { SQ } \\
\text { individuels }\end{array}$ & Autres & Aucun & Total \\
\hline Innovation produit & $47,9 \%$ & $42,9 \%$ & $25 \%$ & $30 \%$ & $39,1 \%$ \\
\hline Innovation procédé & $24,4 \%$ & $23,8 \%$ & $16,1 \%$ & $17,1 \%$ & $20,9 \%$ \\
\hline Innovation emballage & $33,3 \%$ & $36,5 \%$ & $26,8 \%$ & $17,9 \%$ & $27 \%$ \\
\hline Innovation organisationnelle & $32 \%$ & $14,3 \%$ & $28,6 \%$ & $15 \%$ & $23,9 \%$ \\
\hline
\end{tabular}

Si nous nous intéressons maintenant aux modes de gestion des signes de qualité, collectif versus individuel, il apparaît que les signes de qualité gouvernés collectivement sont plus dynamiques que ceux gérés individuellement, du point de vue de l'innovation produit et de l'innovation organisationnelle. Sur les autres composantes de l'innovation, les comportements innovateurs des entreprises sous CCP et $A B$, signes individuels, diffèrent. Le signe AB apparaît comme nettement plus innovateur que le signe $\mathrm{CCP}$, qui se situe en dessous de la moyenne de l'échantillon, sauf sur l'innovation produit. Si l'on agrège les signes de qualité collectifs - AOC, autres indications géographiques et Label rouge -, d'une part, et les signes individuels - CCP, AB -, d'autre part (tableau 6), on constate que les premiers sont plus innovants que les seconds sur toutes les dimensions de l'innovation à l'exception de l'innovation emballage. Ainsi, à titre d'illustration, près de $48 \%$ des entreprises sous signe de qualité collectif ont innové contre $43 \%$ des entreprises sous signe individuel. Dans le cas de l'emballage, c'est probablement le signe individuel $A B$ qui détermine la meilleure performance des signes individuels en matière d'innovation. Or celui-ci est majoritairement représenté par le secteur fruits et légumes, ainsi que produits divers et produits d'origine animale, qui montrent de bonnes performances, en termes d'innovation emballage (voir l'histogramme 3).

On notera également la faible performance en innovation organisationnelle des signes de qualité individuels.
Si ces résultats permettent de conclure à un lien entre les signes de qualité et l'innovation, nous savons par ailleurs que le comportement d'innovation est très lié à la taille de l'entreprise et au secteur d'activité, comme nous l'avons montré plus haut. Nous avons donc cherché à comparer l'effet de certains types de signes de qualité sur les différentes dimensions de l'innovation en présence des variables taille et secteur d'activité.

Les variables dépendantes étant dichotomiques $(\mathrm{Y}=0, \mathrm{Y}=1)$, la régression logistique est la méthode la plus pertinente (Greenan et Walkowiak, 2004) ${ }^{11}$. Il s'agit de déterminer les variables qui influent sur la probabilité d'une entreprise d'innover ou pas dans les 4 types d'innovation.

Nous avons donc introduit dans un modèle de régression logistique (par la méthode ascendante pas à pas de Wald), la taille, la filière, le type de signe de qualité, collectif et territorialisé. Les résultats sont donnés dans le tableau 7.

Nous constatons à l'examen de ce tableau que globalement le taux de bonne prédiction est satisfaisant, ce qui témoigne de la pertinence du modèle. Nous retrouvons un effet taille de l'entreprise significatif dans les différentes dimensions de l'innovation ce qui est en cohérence avec la littérature, alors que l'effet sectoriel n'apparaît que dans le cas de l'innovation organisationnelle.

11. Greenan N., Walkowiak E. (2004). Informatique, organisation du travail et interactions sociales. Document de travail, $\mathrm{n}^{\circ} 31$. 
Tableau 7. Effet des types de signes de qualité sur la propension à innover

\begin{tabular}{l|c|c|c|c} 
& Innov. produit & Procédé & Emballage & Organisationnelle \\
\hline Constante & 0,27 & $-0,66$ & $-0,16$ & 0,67 \\
\hline Effet taille & $0,43^{* *}$ & $0,38^{* *}$ & $0,51^{* *}$ & $0,56^{* *}$ \\
\hline Effet secteur & ns & ns & ns & $0,30^{* *}$ \\
\hline Signe territorialisé & ns & ns & ns & ns \\
\hline Signe collectif & $0,91^{* *}$ & $0,64^{* *}$ & $1,0^{* *}$ & $0,57^{* *}$ \\
\hline$\%$ bonne prédiction & 65 & 79 & 74 & 77 \\
\hline
\end{tabular}

* *Significatif au seuil de $1 \%$

En ce qui concerne l'effet des deux types de signes de qualité, on constate que les signes de qualité territorialisés n'ont aucun effet sur la probabilité d'innover des entreprises, alors que les signes collectifs influencent positivement la propension à innover. Les signes de qualité collectifs non seulement semblent corrélés aux comportements d'innovation, mais apparaissent comme des facteurs influençant positivement ces comportements

\section{Discussion des résultats}

Le premier point à souligner est que l'engagement dans une procédure de qualification, quelle qu'elle soit, est le plus souvent associé à un comportement dynamique en matière d'innovation. S'il est difficile, dans un premier temps, d'affirmer l'existence d'une relation de causalité, on peut toutefois constater un comportement global dynamique, où innovation et adoption de signes de qualité vont de pair, confirmant, au niveau de l'entreprise, les affirmations d'Allaire et Sylvander (op. cit.) et de Touzard (op. cit.). Cependant, des nuances doivent être apportées, en fonction des modes de gouvernance des signes de qualité.

1. Tout d'abord, nous avons constaté que les signes de qualité non territorialisés se situent systématiquement en première place sur toutes les dimensions de l'innovation. Mais il convient de distinguer les différents signes. Le Label rouge se situe presque systématiquement en première position. C'est donc lui qui induit cette primauté. Le signe $\mathrm{CCP}$, quant à lui, est généralement parmi les moins inno- vants, $\mathrm{AB}$ se comportant différemment selon les dimensions de l'innovation. Pourtant, nous avions classé a priori la $\mathrm{CCP}$ comme étant, avec $\mathrm{AB}$, le moins contraignant des signes de qualité, en tant que signe non territorialisé et individuel. Il semble ainsi difficile de conclure que les signes non territorialisés sont systématiquement plus innovants que les signes territorialisés, autrement dit qu'un mode de gouvernance territorialisé des signes de qualité représente une contrainte pour l'innovation.

2. Il apparaît ensuite que la dimension collectif/individuel est plus importante que la dimension territorialisé/non territorialisé dans les comportements d'innovation, mais dans le sens inverse de notre hypothèse de départ. En effet, en dehors du signe $A B$, certes situé en première place pour l'innovation emballage, et en seconde pour l'innovation procédé, mais en mauvaise position pour les autres dimensions de l'innovation, les signes gouvernés collectivement sont associés aux comportements les plus innovateurs. Ce résultat peut être mis en relation avec les différentes approches du processus d'innovation, qui est représenté comme un processus collectif, fondé sur des interactions entre acteurs. Certes, la démarche collective, s'appuyant sur des négociations entre acteurs aux intérêts parfois divergents, entraîne, au niveau individuel, des contraintes. Mais elle conduit à une dynamique globale en matière d'innovation, qui se répercute positivement au niveau de chaque entreprise. 
De plus, la régression logistique réalisée fait apparaître, parmi les trois groupes de variables testées, l'influence de l'engagement dans un signe de qualité gouverné collectivement sur les comportements d'innovation comme la plus importante.

Ces résultats confirment l'importance de certaines formes de proximité dans le processus d'innovation. À travers les signes collectifs non territorialisés, ce sont les proximités institutionnelle et organisationnelle qui sont mises en avant, tandis que la proximité géographique, sans être exclue, est de moindre importance. Nous rejoignons ainsi Rallet et Torre (op. cit.) ainsi que Martin et al. (2006), qui accordent une influence prépondérante à la proximité organisationnelle et institutionnelle par rapport à la proximité géographique dans le cadre des processus d'innovation des entreprises, particulièrement des entreprises agroalimentaires. La notion d'action collective, voire de stratégie collective, apparaît prépondérante par rapport au lien géographique au territoire qui, dans le cas des signes de qualité territorialisés, constitue le fondement même de la construction des règles de l'action collective.

\section{Conclusions}

Il ressort des premiers résultats de l'enquête réalisée en région Languedoc-Roussillon qu'un mode de gouvernance collectif des signes de qualité est le gage d'un dynamisme plus important en matière d'innovation. Cette dimension semble plus importante que l'absence de lien avec le territoire, même si cet aspect n'est pas totalement négligeable. Ce résultat peut être rapproché des observations empiriques réalisées par Montaigne et Cadot (op. cit.) au niveau de la filière vin, où il apparaît que les caves coopératives, par exemple, sont plus innovantes que les caves particulières. Toutefois, dans la mesure où des signes non territorialisés apparaissent systématiquement comme les plus innovants sur toutes les dimensions de l'innovation, on peut se demander si les pro- cédures de spécification des produits liés au territoire, appuyés sur la tradition, n'aboutissent pas à une standardisation progressive des produits et des procédés, ce qui peut conduire à une perte d'abord de biodiversité (Bérard et Marchenay, 2004) et ensuite de certaines des caractéristiques qui participent à la formation de l'identité des produits de terroir dans les représentations des consommateurs (Aurier et al, 2004) à savoir la variabilité des produits selon le producteur (le savoir-faire, la personnalité de chaque producteur doivent être retrouvés).

Ce travail exploratoire soulève toutefois plusieurs questions. Ainsi, la date d'engagement dans les démarches qualité n'a pas été précisée dans les questionnaires d'enquête. Or l'engagement dans une démarche qualité est un processus, susceptible de générer des innovations. On peut se demander si les innovations ne sont pas plus fréquentes lorsque la démarche est récente que lorsqu'elle a débuté depuis plus longtemps. Des études de cas longitudinales semblent nécessaires.

La seconde limite de cette recherche tient à la sur-représentation de la filière viti-vinicole dans l'échantillon total (45\% du nombre d'entreprises constituant près de $95 \%$ des AOC et des autres dénominations d'origine), même si ce pourcentage correspond à la réalité régionale. Ce qui en fait plus une étude sur la filière vin qu'une étude sur l'agroalimentaire en général, et peut expliquer l'absence d'effet sectoriel observé dans la régression logistique. Élargir la recherche à d'autres secteurs permettra d'améliorer la validité externe des résultats. En tout état de cause, la variable sectorielle ne peut être négligée, non seulement du fait de son intervention dans la gouvernance des signes de qualité, mais également parce que les comportements d'innovation semblent différents selon les secteurs.

Enfin, avant de conclure définitivement sur le lien entre gouvernance des signes de qualité et comportements d'innovation, une évaluation comparée des performances des entreprises en fonction des différents signes de qualité semble nécessaire. 


\section{RÉFÉRENCES BIBLIOGRAPHIQUES}

Agreste Languedoc-Roussillon (2004). La sphère agroalimentaire revisitée en 2003, Mutation, recentrage, innovation : l'agroalimentaire régional résiste à la crise. Agreste, janvier, $12 \mathrm{p}$.

Allaire G., Sylvander B. (1997). Qualité spécifique et systèmes d'innovation territoriale. Cahiers d'économie et de sociologie rurales, $\mathrm{n}^{\circ} 44$, p. 29-59.

Akrich M., Callon M., Latour B. (1988). À quoi tient le succès des innovations. Deuxième épisode : l'art de choisir les bons porte-paroles. Annales des Mines, Gérer et comprendre, septembre, $\mathrm{n}^{\circ} 12$, p. $14-29$

Ambiaud E. (2007). Le marché comme ressort de l'innovation agroalimentaire. Agreste Primeur, mars, n¹92, 4 p.

Aurier Ph., Fort F., Sirieix L. (2004). Les produits de terroir pour le consommateur : première proposition d'identification des sources perçues et des associations au terroir. Congrès de l'AFM, Saint Malo, 5-7 mai.

Ayerbe C. (2006). Innovations technologique et organisationnelle au sein de PME innovantes : complémentarité des processus, analyse comparative des mécanismes de diffusion. Revue Internationale PME, vol.19, $\mathrm{n}^{\circ} 1$, p. 9-34.

Bhattacharya M., Bloch H. (2004). Determinants of Innovation. Small Business Economics, ${ }^{\circ} 22$, p. 155-162.

Bérard L., Marchenay Ph. (2004). Les produits de terroir entre cultures et règlements. Paris, CNRS Éditions, 229 p.

Belotti C., Temri L. (2004). Chapitre 8 : Stratégies d'innovation, compétitivité, emploi. In Perez R., Brabet J., Yami S. (Eds.), «Management de la compétitivité et emploi », Paris, L'Harmattan, p. 201-220.

Casabianca F., Valceschini E. (1996). La qualité dans l'agroalimentaire : émergence d'un champ de recherche. Paris, INRA Éditions, 344 p.
Crozier M., Friedberg E. (1977). L'acteur et le système. Paris, Éditions du Seuil, 1977.

Delfosse C., Letablier M.-T. (1995). Genèse d'une convention de qualité : les appellations d'origine. In G. Allaire et R. Boyer (Eds), «La grande transformation de l'agriculture », Paris, Economica. Divry C., Dubuisson S., Torre A. (1998). Compétences et formes d'apprentissage : pour une approche dynamique de l'innovation. Revue Française de Gestion, 118, p. 115-127.

Foray D. (1996). Diversité, sélection et standardisation : les nouveaux modes de gestion du changement technique. Revue d'Économie industrielle, $\mathrm{n}^{\circ} 75$, p. 257-274.

Fort F., Fort F. (2006). Diminuer les risques d'échec lors de la mise en œuvre des « changements packagés ». Revue Française de Gestion, ${ }^{\circ} 166$, p. 51-68.

Fort F., Rastoin J.-L., Temri L. (2005). Les sources de production d'innovations en PME/TPE agroalimentaire. Revue Internationale PME, vol. 18, $\mathrm{n}^{\circ}$ 1, p.47-72. Freel M.-S. (2003). Sectoral patterns of small firm innovation, networking and proximity. Research Policy, $\mathrm{n}^{\circ} 32$, p. 751-770.

McAdam R., Armstrong G., Kelly B. (1998). Investigation of the relationship between total quality and innovation: a research study involving small organisations. European Journal of Innovation Management, vol.1, $\mathrm{n}^{\circ} 3$, p. 139-147. Martin M., Tanguy C., Albert P. (2006). Capacité d'innovation des entreprises agroalimentaires et insertion dans les réseaux : le rôle de la proximité organisationnelle. Économie Rurale, ${ }^{\circ} 292$, p. 35-49.

Mollard A. (2001). Qualité et développement territorial. Un outil d'analyse : la rente. Economie Rurale, n 263 , p. 16-34. 
Montaigne E., Cadot J. (2006). L'innovation dans la filière vitivinicole du Languedoc Roussillon. In « Dynamiques des entreprises du Languedoc Roussillon : évolutions 1998-2003 », Montpellier UMRMOISA, Cahier de recherche $\mathrm{n}^{\circ}$ 03-2006, p. 89-101.

Nonaka I., Takeuchi H. (1997). La connaissance créatrice - La dynamique de l'entreprise apprenante. De Boeck Université, 303 p.

OCDE (2005). Manuel d'Oslo. Principes directeurs pour le recueil et l'interprétation des données sur l'innovation. Paris, OCDE, troisième édition.

Pamukçu T., Cincera M. (2001). Analyse des déterminants de l'innovation technologique dans un nouveau pays industrialisé : une étude économétrique sur données d'entreprises dans le secteur manufacturier turc. Économie et Prévision, $\mathrm{n}^{\circ}$ 150/4-5, p. 139-158.

Pavitt K. (1984). Sectoral patterns of technical change : towards a taxonomy and a theory. Research Policy, n ${ }^{\circ} 3$, p. 343373.

Pecqueur B., Zimmermann J.-B. (2003). Les fondements d'une économie de proximités. In Pecqueur B. Zimmermann J.-B., (Eds) «Économie de proximités », Paris, Lavoisier, p. 13-41.

Perez R. (2003). La gouvernance de l'entreprise. Paris, La Découverte, coll. Repères, $\mathrm{n}^{\circ} 358$.
Prajogo D.I., Sohal A.S. (2001). TQM and innovation : a literature review and research framework. Technovation, $\mathrm{n}^{\circ} 21$, p. 539-558.

Rallet A., Torre A. (Eds) (2007). Quelle proximité pour innover? Paris, L'Harmattan, $221 \mathrm{p}$.

Roux P. (1999). Nouveau management et technologie de l'information vont de pair dans les IAA. Agreste, Les Cahiers, $n^{\circ} 37$, p. 29-35.

Sylvander B., Marty F. (2000). Logiques sectorielles et territoriales dans les AOC fromagères : vers un compromis par le modèle industriel flexible ? Revue d'Économie Régionale et Urbaine, $\mathrm{n}^{\circ} 3$, p. 501-518.

Tirole J. (1996). A theory of collective reputation (with applications to the persistence of corruption and to firm quality). Review of Economic Studies, 63, p.1-22. Touzard J.-M. (2000). Coordinations locales, innovation et régulation : l'exemple de la transition « vins de masse » - « vins de qualité » en Languedoc-Roussillon. Revue d'Économie Régionale et Urbaine, $\mathrm{n}^{\circ} 3$, p. 589-604.

Valceschini E., Nicolas F. (1995). La dynamique économique de la qualité agroalimentaire. In F. Nicolas, E. Valceschini (Eds), "Agro-alimentaire, une économie de la qualité », Paris, INRA.

Vincenau M. (2003). Innover pour le marché. Agreste Primeur, avril, n 122. 